\title{
Anaximander, Patrokles, oder vielleicht Eudoxos? Resümee einer Deutungsgeschichte des sogenannten Trierer Anaximander-Mosaiks vor dem Hintergrund der biographischen, doxographischen und archäologischen Zeugnisse
}

\section{Georg Wöhrle}

Universität Trier,

Universitätsring 15, 54286, Trier, Deutschland; woehrle@uni-trier.de

For citation: Georg Wöhrle. Anaximander, Patrokles, oder vielleicht Eudoxos? Resümee einer Deutungsgeschichte des sogenannten Trierer Anaximander-Mosaiks vor dem Hintergrund der biographischen, doxographischen und archäologischen Zeugnisse. Philologia Classica 2018, 13(2), 200-212. https://doi.org/10.21638/11701/spbu20.2018.201

A fragment of mosaic dating to the second century CE, the shard of a larger composition, discovered in 1898 on an excavation site of a Roman domus in Trier, and currently part of the collection of Rheinisches Landesmuseum, is well preserved, meticulously executed and not untouched by genius. A bald philosopher with a full grey beard rimming his face is seated holding in his lap what is very likely to be a sundial. He is turned halfway to the front, aiming his gaze up over his right shoulder. It is an easy guess, and on initial publication the then director of Landesmuseum Emil Krüger tentatively identified the figure as Anaximander. Over a hundred years on, the opinion has not changed much. Sceptical minds have been spurred by the lack of evidence on Anaximander to go on to identify the figure with a certain sculptor $\mathrm{Pa}$ trocles, the inventor, according to Vitruvius, of a kind of sundial called pelecinum. It is unlikely that an artist would draw inspiration from such an obscure person. Better still, a philosopher a cut above Anaximander, Eudoxus, could have served as a prototype, especially since he had also built a sundial called arachne. As we know from Vitruvius, it was of a different shape, but one would not expect an artist to be true to hard fact. However long we strain our eyes to grasp the features of Eudoxus and Anaximander in the 'mosaics of philosophers' from Pompeii, the Roman Anaximander in an inscribed relief, or other works of similar type, any secure attribution will still be out of our reach. The reception of Anaximander in the classical culture will not have been much different: it was he who came down to late Roman art as the inventor of 
sundial. Had we possessed the yet lacking parts of the mosaic, we would probably have seen other inventors. A shadow of doubt is cast over the idea of K. Schefold that Anaximander gazes skywards comprehending ,the coming to being and destruction of heavenly bodies". Clearly, an inventor of sundial would rather be watching the sun.

Keywords: Anaximander, Roman mosaic from Trier, Eudoxus, sculptor Patrocles, portraits of ancient philosophers.

Das sogenannte Trierer Anaximander-Mosaik aus dem zweiten Jahrhundert n. Chr. (Abb. 1), das gegen Ende des 19. Jahrhunderts in der Johannisstraße 23 der Stadt gefunden wurde und nur Teil eines einstigen Gesamttableaus bildete, ${ }^{1}$ ist dank seiner hohen Qualität von einiger Bekanntheit. ${ }^{2}$ Obwohl wir bei dem Trierer Mosaik keine Inschrift haben wie bei einem Marmorrelief aus dem Museo Nazionale Romano, die den Philosophen einigermaßen eindeutig identifizierte (Abb. 3), scheint eine solche Identifikation, vor allem aufgrund der Sonnenuhr in der Hand der dargestellten Person, für die meisten Fachleute zumindest plausibel zu sein. So heißt es bereits in der ersten kurzen Fundbeschreibung von 1909 durch den damaligen Museumsdirektor Emil Krüger: „Sitzender Gelehrter mit Sonnenuhr, vielleicht Anaximander, als erster Verfertiger von Sonnenuhren in Griechenland, gef. 1898 in der Johannisstraße, von besonders feiner Mosaikarbeit“" 3 Wolfgang Binsfeld, stellvertretender Direktor des Landesmuseums, schrieb in den siebziger Jahren des vergangenen Jahrhunderts dann schon: „Es ist Anaximander, der Erfinder der Sonnenuhr". Eine Ansicht, die bis heute, wie gesagt, wesentlich vorherrscht. Die Frage wäre dann nur, welche Vorstellung man in römischer Zeit in Trier mit Anaximander verband. Binsfeld meinte jedenfalls, dass „Anaximanders Weltschau [...] dem Schöpfer und dem Besteller des Trierer Mosaiks [...] sicher ebenso wenig gegenwärtig [war], wie dem Besucher des [Rheinischen] Landesmuseums, für sie war er einfach ein Weiser, der Erfinder der Sonnenuhr" 5

Doch sehen wir zunächst einmal, was wir heutzutage an im engeren Sinne biographischen Nachrichten über Anaximander aus der Antike erhalten haben, blicken wir gewissermaßen auf das literarische Bild des Philosophen. Der Befund ist ziemlich dürftig. Selbst Diogenes Laertios' Biographie (II 1 und 2) ist kaum als solche zu bezeichnen. Sie gehört zu den kürzeren bzw. kürzesten in seinem Werk und bietet auf einer Seite gerade einmal den Hinweis auf die Herkunft aus Milet und den Vater Praxiades sowie das Lebensalter. Im zweiten Jahr der 58. Olympiade (547/546) sei er 64 Jahre alt gewesen und kurz danach gestorben. Einzig eine etwas merkwürdige Anekdote beschließt den biographischen Zusammenhang. Er soll, so schreibt Diogenes, wegen seines Gesanges von Kindern verspottet worden sein und, als er davon erfuhr, gesagt haben: „Dann muss ich

1 Siehe dazu die Beiträge von Krüger 1909b, Parlasca 1959, Binsfeld 1977, Schefold 1997, 382-383 mit Abb. 250 und Hoffmann, Hupe und Goethert 1999, 112 (mit genauen Angaben zu den Fundumständen; die Verf. vermuten, dass das Mosaik einst Teil einer ursprünglichen Vierfelder-Gesamtkomposition gewesen sein könnte).

${ }^{2}$ Es findet merkwürdigerweise in der recht umfassenden Sammlung von Jörn Lang (2012) zur Rezeption griechischer Dichter und Denker in der römischen Lebenswelt im Gegensatz zu einem römischen Marmorrelief keine Erwähnung. Ebenfalls nicht in Winter 2013.

${ }^{3}$ Krüger 1909a, 136. Eine ausführliche Beschreibung des Fundes und zugleich eine Abbildung des noch nicht ergänzten Mosaiks bei Krüger 1909b, mit Tafel III 1.

${ }^{4}$ Binsfeld 1977, 130.

${ }^{5}$ Binsfeld 1977, 131. Über die erste Annahme lässt sich natürlich nur spekulieren. 
meinen Gesang um der Kinder willen verbessern." Dazwischen streut Diogenes Bemerkungen eher doxographischer Art ein. Zum Apeiron als Prinzip, zur Position der Erde im Kosmos, zur Astronomie und Geographie. Die ,Erfindung' des Gnomons und von Stundenanzeigern, die uns noch im Weiteren beschäftigen wird, wird ebenfalls erwähnt. Wenn wir die zu Anaximander aus der Antike überlieferten Nachrichten durchmustern, erweitern sich die biographischen Angaben nicht wesentlich. Hervorgehoben wird von Themistios der ,Mut', als erster eine schriftlich verfasste Abhandlung über die Natur zu veröffentlichen. ${ }^{6} \mathrm{Ob}$ in Favorins Universalgeschichte, eines Vertreters der sogenannten Zweiten Sophistik aus dem zweiten Jahrhundert n. Chr., oder in Apollodors Chronika, die Diogenes beide als Quelle zitiert, wesentlich mehr zu finden war, können wir natürlich nicht sagen. In späteren Büchern erwähnt Diogenes noch Diodor von Ephesos, der eine Schrift über Anaximander verfasst haben soll, und darin schrieb, dass Empedokles diesen nachgeahmt habe, indem er einen pathetischen Schwulst praktizierte und eine erhabene Kleidung anlegte $\left(8,70=\operatorname{Ar} 94^{7}\right)$. Möglicherweise handelt es sich aber um eine Verwechslung, und diese Schrift betraf eigentlich Anaxagoras. ${ }^{8}$ Weiter zitiert Diogenes auch noch (9,18=Ar 95), allerdings in der Xenophanes-Biographie, den Peripatetiker (?) Sotion, nach dem Xenophanes ein Schüler Anaximanders gewesen sei. Dass es natürlich noch weitere biographische Informationen gegeben haben muss, beleuchten eben die vorhin schon zitierte Anekdote bei Diogenes Laertios zu Anaximanders schlechtem Gesang und eine weitere Anekdote, die bei Cicero (Div. 1, 50, 112=Ar 27) und bei Plinius (HN 2, $191=$ Ar 42) erhalten ist. Danach hatte Anaximander, der auch hinsichtlich des Gnomons, wie gleich noch zu sehen, mit Sparta in Verbindung steht, die Spartaner einst gewarnt, ihre Stadt und ihre Häuser zu verlassen und in Waffen auf dem Feld im freien zu schlafen, weil ein Erdbeben drohe, das dann auch tatsächlich eintrat.

Viel mehr an spezifischen Angaben zu Anaximanders Lebensumständen ist aber, wie gesagt, aus der Antike nicht erhalten. Das mag auch dem Zufall der Überlieferung geschuldet sein, denn selbst von Anaximanders Nachfolger Anaximenes sind (bei Diogenes Laertios 2, 4-5) wenigstens zwei fiktive Briefe an Pythagoras erhalten, die vielleicht auf einen romanhaften Zusammenhang verweisen. Dass er aber als ein namhafter Gelehrter ${ }^{9}$ auch in hellenistischer Zeit noch bekannt war, geht aus einer Liste der Bibliotheksbestände des Gymnasiums von Tauromenion in Sizilien aus dem 2. Jh. v. Chr. hervor, in der Anaximander erwähnt ist (Ar 23). ${ }^{10}$

Wenn freilich die biographischen Angaben so spärlich fließen, und vielleicht auch in der Antike nicht so furchtbar viel davon bekannt gewesen ist - jedenfalls nicht derart, dass es sich in einer bildlichen Darstellung materialisieren ließe -, stellt sich die Frage, wofür, wenn überhaupt, Anaximander in einer notabene gebildeten Schicht überhaupt bekannt gewesen ist. Der Großteil der engeren doxographischen Überlieferung betrifft natürlich das berühmte ,Prinzip', seine kosmogonischen bzw. kosmologischen Theorien

${ }^{6}$ Rede 26, 317C, Ar 120.

7 Wöhrle 2012, zitiert als Ar (Anaximander) bzw. As (Anaximenes).

8 Siehe dazu die kritischen Apparate in den Ausgaben von Marcovich 2012 bzw. Dorandi 2013.

9 Auf dem Marktplatz in Milet wurde ein Teil einer Korenstatue aus etwa der Mitte des sechsten Jahrhunderts v. Chr. gefunden, auf der sich der Name Anaximanders (Weihinschrift?) findet. Siehe hierzu Darsow 1954.

10 Siehe Blanck 1997. Merkwürdig erscheint, dass in der Aufzählung der Statuen des ZeuxipposGymnasiums von Konstantinopel bei Christodor (Ekphrasis 50-51=As 176) zwar Anaximenes, nicht aber Anaximander erwähnt wird. 
und gelegentlich seine ,Evolutionstheorie' des Menschen. Aber es ist eindeutig Anaximanders angebliche Entdeckung des Gnomons bzw. der Gnomonik, die die Aufmerksamkeit auch nicht im engeren Sinne doxographischer Autoren auf sich gezogen haben. Anaximander kann vielleicht mit einigem Recht als der erste Metaphysiker des Abendlandes bezeichnet werden, ${ }^{11}$ doch die Frage nach dem Prinzip aller Dinge, die er mit dem Hinweis auf das Unendliche oder Grenzenlose, das Apeiron, die infinitas naturae, wie es Cicero nennt, ${ }^{12}$ beantwortete, war sicher eher eine Sache philosophischer Diskussionen als populärwissenschaftlicher Diskurse. Dasselbe gilt für seine Spekulationen über den Aufbau des Universums, das sich nach Anaximander, nachdem es in einer Art ,Big Bang' entstanden war, ${ }^{13}$ in einem komplexen geometrischen Symmetrieverhältnis entfaltete. Allenfalls die Nachricht seiner Pionierleistung als Geograph, er soll als erster eine Karte der bewohnten Welt gezeichnet haben, so Agathemeros, ${ }^{14}$ dringt jenseits philosophisch orientierter Doxographien auch in die spezielle geographische Literatur ein. ${ }^{15}$ Die angebliche Erfindung des Gnomons jedoch, die in unserem Zusammenhang vor allem interessiert, wird bei mehreren Autoren erwähnt, u. a. auch in der kurzen schon erwähnten Biographie des Diogenes Laertios. Es heißt dort: ${ }^{16}$ „Er [Anaximander] erfand auch als erster den Gnomon und stellte ihn, der Wenden und Tag-und-Nacht-Gleichen angibt, bei den Sonnenuhren (epì tōn skiothērōn) in Sparta auf, wie Favorinus in seiner Universalgeschichte sagt. Auch Stundenanzeiger (hōroskópia) hat er verfertigt". Als nächster erwähnt Eusebios von Kaisareia in seiner Praeparatio Evangelica ${ }^{17}$ die Sache mit dem Gnomon, wobei bemerkenswert ist, dass dadurch Anaximander von den beiden anderen Milesiern, Thales und Anaximenes gewissermaßen spezifisch unterschieden wird. Die noch späteren Nachrichten brauchen uns hier nicht weiter zu interessieren, abgesehen davon, dass auch in der Suda ${ }^{18}$ Anaximander einerseits unter dem Namenseintrag als Entdecker des Gnomons hervorgehoben ist und andererseits, was vielleicht noch bedeutsamer, im Eintrag ,Gnomon' sogleich auf den Milesier verwiesen wird. Etwas quer steht nur die Nachricht in der Naturgeschichte des älteren Plinius, wonach Anaximenes aus Milet, der Schüler des Anaximander, die Berechnung der Schatten, die man Gnomonik nenne, erfunden habe. Er habe auch als erster in Sparta eine Uhr gezeigt, die man ,Skiotherikon' nenne. ${ }^{19}$ Es liegt hier allerdings mit großer Gewissheit nahe, an eine Verwechslung von Seiten des Plinius zu denken, da seine Nachricht ansonsten allen anderen in der antiken Überlieferung widerspricht und die lateinische Formulierung: primusque horologium, quod appellant sciothericon, Lacedaemone ostendit ja in den eben aufgeführten Nachrichten bei Diogenes Laertios in eher unverstandener Weise wiederauftaucht.

Was nun die Sache selbst betrifft, die Entdeckung des Gnomons und die Erfindung der Sonnenuhr, so müssen wir hier nicht viele Worte darüber verlieren, zumal darüber

\footnotetext{
11 So Couprie 2011, 97.

12 Lucullus 37, 118 (=Ar 28).

13 Mansfeld, Primavesi 2011, 57.

14 Geographie 1, 1-4=Ar 47.

15 Siehe die Similien (Pionier der Geographie/Schrift) zu Ar 31.

16 2, 1 (Ar 92).

$1710,14,11-12=$ Ar 102 .

18 Lexikon Alpha 1986 (= Ar 237, vgl. Ar 239, 240).

19 NH 2, 187=Ar 41. Zur Unsicherheit späterer Autoren bezüglich der Frage, was denn nun Anaximander genau entdeckt bzw. eingeführt hat, vgl. Winter 2013, 114 und 143.
} 
schon eine überaus reiche Literatur existiert. ${ }^{20}$ Fraglos ist, dass Anaximander den Gnomon allenfalls nach Griechenland bzw. Sparta als erster eingeführt haben kann; denn die Gnomonik war, wie bereits Herodot wusste, schon den Babyloniern bekannt. ${ }^{21}$ Diskutiert wird, vor welchem theoretischen Hintergrund Anaximanders Sonnenuhr, wenn er sie denn aufgestellt hat, funktionierte. Aber das sind Fragen der Rekonstruktion, während es uns hier um eine Frage der Rezeption geht. Wie auch immer wurde, wie gesehen, Anaximander als Entdecker der Gnomonik identifiziert und auch in Rom bekannt, und es leuchtet jedenfalls ein, dass man am ehesten mit Anaximander, dem ,Gnomoniker' einen Markenkern fände, der sich unter Umständen auch in einer künstlerischen Darstellung zum Ausdruck bringen ließe.

Betrachten wir also das Mosaik etwas genauer, bei dem es sich laut Klaus Parlasca ${ }^{22}$ „um das Eckstück eines größeren Bodens mit mehreren Bildfeldern zu handeln" scheint (s. Abb. 1). Im Zentrum der Darstellung, der „noch vor 200 n. Chr. entstandenen Kopie eines hochhellenistischen Bildes", wie Karl Schefold schreibt, ${ }^{23}$ sehen wir einen älteren Mann mit Halbglatze und „voluminösen Bartlocken “24. Der kräftige, gebräunte Oberkörper wird durch das geöffnete Gewand (Himation) sichtbar. Der so deutlich als griechischer Denker bzw. Philosoph gekennzeichnete Mann ${ }^{25}$ wendet den Blick nach rechts (bzw. vom Betrachter aus nach links) oben, vielleicht einer anderen Person bzw. dem Betrachter zu. In der linken Hand hält er einen Gegenstand, der auf den ersten Blick wie ein riesiger Schmetterling aussieht, in der rechten eine Art Griffel oder radius, mit dem er in der Manier eines Lehrenden auf diesen Gegenstand deutet. Bei diesem Gegenstand handelt es sich um eine spezifisch geformte Sonnenuhr, wobei der in ihrer Mitte oben angebrachte kleine senkrecht stehende Stab einen Gnomon darstellt (Abb. 2). Diese Legende wird noch dadurch unterstrichen, dass die linke Seite der Sonnenuhr beschattet erscheint wie ebenso der Gnomon einen Schatten in die Felder der rechten Seite wirft. Dass es zur Entstehungszeit des Mosaiks vielfältige Sonnenuhren allerorten gab, ist selbstverständlich. Erst in jüngerer Zeit ist jedoch, soweit ich sehe, die besondere Form und damit der spezielle Typus Sonnenuhr identifiziert worden. Sie wird von Marcus Cetius Faventinus, einem Fachschriftsteller aus dem dritten Jahrhundert n. Chr. in seiner Abhandlung über Sonnenuhren beschrieben ${ }^{26}$ und als pelecinum benannt ${ }^{27}$. Diese in Form einer Doppelaxt (pélekys) geformte Sonnenuhr war laut diesem Autor aus zwei Marmor- bzw. Steinplatten zusammengesetzt, die jeweils am oberen Ende breiter, am unteren schmaler waren. Diese Platten waren ihrerseits ,jeweils mit 5 ausgerichteten Linien markiert, so dass sie eine

20 Siehe hierzu Couprie 2011, 28-36 mit weiterer Literatur.

21 Historien 2, 109.

22 Parlasca 1959, 29.

${ }^{23}$ Schefold 1997, 382.

24 Von den Hoff 1994, 159.

25 Siehe Lang 2012, 22-23 zur Typologie römischer Darstellungen griechischer Dichter bzw. Denker. Dass ein Vorsokratiker dargestellt wäre, ist prinzipiell unproblematisch. „Statuen vorsokratischer Philosophen existierten bereits seit dem 4. Jh. [...]. Im 2. Jh. ist ein wachsendes Interesse an Darstellungen vorsokratischer Philosophen zu beobachten“ (von den Hoff 1994, 159-160).

26 De architectura privata 29, 2 (de horologii institutione).

27 Bzw. pelignum mit den Codices. Erste zweisprachige deutsche Ausgabe von Kai und Christiane Brodersen (2015). Vgl. auch Pattenden 1979, Schaldach 2001, 33-34, Traversari 1991, bes. 69. Vgl. auch: Severino (internet-Publikation) und den Beitrag von Brodersen 2016, bes. 75, Anm. 15 zu pelignum versus pelecinum. 
Ecke bilden, welche die (sonst fehlende Linie für die) 6. Stunde zeichnen soll“. ${ }^{28}$ Diese und die noch folgende Beschreibung kann man, bei Einräumung einiger künstlerischer Abstraktion, in dem Mosaik etwa wiederfinden. ${ }^{29}$

Nun hatte man ja, wie eingangs bemerkt, zwar gleich mit der Entdeckung des Mosaikes an eine Darstellung Anaximanders gedacht. Schon Krüger schrieb, dass „das kleine Relieffragment (Abb. 3) im Thermenmuseum in Rom [...], das nach der Inschrift den Anaximander darstellen soll, [...] mit der hohen Stirn, dem Vollbart, der das Kinn frei lässt, und in der Gewandung einen sehr ähnlichen Typus [gibt] “. ${ }^{30}$ Dem schloss sich Klaus Parlasca an, der in seiner ausführlichen Beschreibung nachdrücklich auf eine „Verwandtschaft" der in Trier dargestellten Figur „in Haaranordnung und Kopfform“ mit derjenigen, inschriftlich als Anaximander bezeichneten, im ebenfalls eingangs erwähnten Relieffragment des Nationalmuseums hinweist und Zweifel daran für eher unbegründet hält „in Anbetracht der fraglos individuellen Züge des Mannes“. ${ }^{31}$ Dieses Relief dient dann im Weiteren in Zusammenhang mit dem Trierer Mosaik Ralf von den Hoff (in einem etwas zirkulären Schluss) ${ }^{32}$ als Bestätigung der Benennung eines rundplastischen, nur in drei Repliken erhaltenen Porträts aus dem zweiten Viertel des 2. Jhs. v. Chr. als Anaximander. Freilich hat man auch gesehen, dass die Pose des Anaximanders auf dem Relief eben anders gestaltet ist, im ,Denkergestus, ${ }^{33}$ und die Figur wahrscheinlich keinen Gegenstand in der Hand hielt. Deutliche Parallelen gibt es zwar sicherlich in der Gestaltung des vollbärtigen Hauptes mit Halbglatze, wobei aber im Mosaik die grübelnde Brauenkontraktion des Denkers fehlt. Überhaupt gilt zu bedenken, dass „sowohl Sonnenuhr als auch Globus [...] polyvalente Bedeutungsträger [sind]. Als spezifische Attribute verweisen sie auf den astronomischen Bereich oder in die Naturwissenschaft und sind gleichzeitig auf einer allgemeineren Ebene als «Bildungsrequisiten» zu verstehen". ${ }^{34}$ So ist Skepsis durchaus nachvollziehbar, auch wenn der Gnomon nun einmal, wie gesehen, bis in die Spätantike hinein das Merkmal des Milesiers war. Gustavo Traversari äußert diese Skepsis entsprechend: ${ }^{35}$ „In sostanza, il riconiscimento di Anassimandro nel mosaico di Treveri si basa soltanto

${ }^{28}$ Marcus Cetius Faventinus (Kai und Christiane Brodersen 2015) ebd. Eine anschauliche Rekonstruktion findet sich bei Auber (internet-Publikation).

${ }^{29}$ Der Trierer Mosaikkünstler hatte allerdings möglicherweise keine genauen Kenntnisse von Sonnenuhren. Jedenfalls verlaufen die sechs (anstelle von fünf) Linien nicht so, wie bei Cetius beschrieben.

30 Krüger 1909b, 16.

31 Parlasca 1959, 29. Die Datierung ist umstritten. Während Schefold 1997, 310 und von den Hoff 1997, 158 für die frühe Kaiserzeit plädieren, argumentiert Blanck 1999, 48 aufgrund der Inschrift für eine Datierung ins zweite Jahrhundert v. Chr. (Lang 2012, 178: Ende 2. - frühes 1. Jh. V. Chr.).

32 Von den Hoff 1994, 155-160, bes. 159, wo die Identifikation des Mosaiks als gegeben genommen und auf dessen Übereinstimmung in der Frisuranlage an der linken Schläfe mit der des Typus der Repliken hingewiesen wird.

33 Blanck 1999, 47. Vgl. Schefold 1997, 310: „Der mit nacktem Oberkörper nach rechts sitzende Weise ähnelt in seinen Zügen denen des Mosaiks in Trier [...], stellt ihn aber in sorgenvollem Sinnen dar [...].“

${ }^{34}$ Lang 2012, 89. Vgl. auch die von Lang ebd. 83, 88-89 aufgeführten Beispiele einer bärtigen Gestalt vor Sonnenuhr auf Glasgemmen bzw. Karneolen (G TypA63 - 67 und G TypD1 - 8: „Erklärende mit radius“). Parlasca 1959, 29, Anm. 2 verweist selbst auf „die ähnliche Darstellung eines Gelehrten mit Sonnenuhr auf einem Mosaik von Morton Farm bei Brading (Insel Wight)“. „The figure may perhaps be intended for Hipparchus" (Morgan 1886, 237). Auch eine von Karl Schefold 1997, 440 vorgelegte Vermutung, wonach es sich bei einer Abbildung auf dem Fragment eines Riefelsarkophages aus der Mitte des 3. Jh. n. Chr. um Anaximander handle, ist von Ewald 1999, 162 zu Recht als nicht zwingend abgelehnt worden: „die geläufige Sonnenuhr jedenfalls vermag die Deutung nicht auf einen bestimmten Philosophen einzuengen“.

35 Traversari 1991, 69, der auch die ikonographischen Parallelen mit dem Relieffragment aus dem Museo Nazionale Romano als zu geringfügig ansieht. 
sul fatto che il vecchio seduto ha un orologio solare in mano e basta“. Traversari schlägt seinerseits vor, ${ }^{36}$ dass es sich bei dem Dargestellten um den Griechen Patrokles handeln könne, den Vitruv ${ }^{37}$ als Erfinder der pelecinum-Uhr ausweist. Dem kann man entgegenhalten, dass es problematisch erscheint, in dem Mosaik eine sonst so unbekannte Figur identifizieren $\mathrm{zu}$ wollen (und gerade doch nur wieder aufgrund des einen Indizes der Sonnenuhr). ${ }^{38}$

Gelangt man hier noch weiter? Emil Krüger hatte seinerzeit bereits auf die beiden Philosophenmosaiken von Pompeji bzw. Neapel und von Sarsina verwiesen: „Die zweite Figur von links auf dem Mosaik von Pompeji (Abb. 4 und 5) ist in Antlitz, Kleidung und Haltung dem Trierer Philosophen ähnlich, die Sonnenuhr [oben auf der Säule] hat dieselbe Form auf dem Bild von Sarsina (Abb. 6). " ${ }^{39}$ Nun ist die Identifikation der Figuren auf den beiden Mosaiken zwar sehr umstritten, doch scheint Anaximander hier eher nicht in Frage zu kommen. Man denkt an die Schule Platons oder eine Darstellung der Sieben Weisen. ${ }^{40}$ Konrad Gaiser ${ }^{41}$ hat in seiner Interpretation die fünfte, unter einer Sonnenuhr sitzende Figur für Eudoxos in Anspruch genommen, den man ansonsten nur noch auf einem allerdings kopflosen Relief in Budapest sicher identifizieren kann. ${ }^{42}$ Auch Eudoxos hat im Übrigen laut Vitruv eine bestimmte Sonnenuhr mit der Bezeichnung arachne erfunden, ${ }^{43}$ die zwar kaum dem auf dem Trierer Mosaik dargestellten Beispiel eines solchen Instruments entspricht. ${ }^{44}$ Man darf aber hier entgegnen, dass es dem Künstler wohl nicht auf eine wissenschaftshistorisch exakte Darstellung ankam. Könnte man infolgedessen bei der Trierer Darstellung, die übrigens in der Gestaltung des Kopfes der fünften Figur aus Neapel (Abb. 7) nicht völlig unähnlich scheint, auch an Eudoxos, eine sicher renommiertere Gelehrtenpersönlichkeit noch als Anaximander, denken? Auf der anderen Seite ist die von Gaiser als ,Eudoxos' vermutete Figur aus dem Neapler Mosaik in ihrem denkerischen Gestus wiederum eher dem römischen Anaximander-Relief vergleichbar. Doch soll diese Diskussion von Vermutungen auf sandigem Grund hier nicht weitergeführt werden.

Letztlich bleibt natürlich nur ein ,non liquet' hinsichtlich der Frage, ob mit der im Trierer Mosaik dargestellten Gestalt Anaximander oder ein anderer Gelehrter gemeint war; die literarischen und ikonographischen Indizien widersprechen einer Zuschreibung zu Anaximander zumindest nicht. Unbeantwortbar ist weiterhin die Frage, was der antike Betrachter in das Bildnis (das für ihn ja in einem uns unbekannten künstlerischen und räumlichen Kontext stand ${ }^{45}$ hineinlas und erst recht, ob der Auftraggeber bzw. Künstler

36 Traversari 1991, 69.

37 De architectura 9, 8, 1.

38 Zudem wäre dann zu erwarten, dass die Sonnenuhr korrekt dargestellt wäre (vgl. oben Anm. 28).

39 Krüger 1909b, 16. Die besten Abbildungen jetzt bei Andreae (2005) 12f. mit Abb. 1 und 2.

40 Vgl. zur Diskussion den Beitrag von Andreae (2005).

41 Gaiser 1980, 62-63 und Schefold 1997, 296.

42 Siehe Blanck 1999, 48 mit Tafel 8.4. Auch bei der Abbildung eines stehenden Gelehrten auf einem Kantharos des Silberschatzes von Berthouville lässt sich an Eudoxos denken. Siehe Schefold 1997, 288 mit Abb. 165 und 166. Vgl. auch ebd. 296 mit Abb. 173.

${ }^{43}$ De architectura 9, 8, 1: arachnen Eudoxus astrologus, nonnulli dicunt Apollonium.

44 Zum möglichen Aussehen dieses Typus'von Sonnenuhr siehe Hannah 2009, 87.

45 „Die Fundstelle liegt nordwestlich des Forums, in dessen Umfeld weitere repräsentative Fußbodenmosaiken (z. B. das Musenmosaik aus der Johannisstraße Nr. 9) zutage kamen [...]. Man wird den Befund wohl nicht überstrapazieren, wenn man das dortige Areal als gehobenes römisches Wohnviertel bezeichnet. Im Fall des Anaximander-Mosaiks fehlen allerdings jegliche konkreten Anhaltspunkte für 
eine Vorstellung von Anaximanders Weltmodell hatte. ${ }^{46}$ Dass die Gnomonik auf der Konnotationsebene für einen sehr gebildeten Betrachter des Mosaiks bzw. der Darstellung auf die weiteren kosmologischen Spekulationen des Vorsokratikers, wenn er denn gemeint wäre, verweist, ist natürlich nicht von der Hand zu weisen, wie ja eben die Forschungsgeschichte seit der Wiederentdeckung des Mosaiks zeigt. Ob man, wie etwa Schefold, ${ }^{47}$ am scheinbar „visionär nach oben gerichteten Blick“ des dargestellten Mannes auch gleich noch an dessen „kühne Vorstellung vom Werden und Vergehen der Himmelskörper“ denken sollte, sei dahingestellt. Vielleicht findet sich ja eines Tages noch ein weiterer ,Mosaikstein, der den Trierer Gelehrten mit der Sonnenuhr ${ }^{48}$ besser verstehen lässt. ${ }^{49}$

\section{Literaturverzeichnis}

Albéri-Auber P. Orologi solari greco-romani. Pelecinum. Available at: http://www.ingauber-meridiane. it/?page_id=484 (16.10.2018)

Andreae B. Das Mosaik der Sieben Weisen aus Sarsina in der Villa Albani in Rom und sein Verhältnis zum Philosophenmosaik aus Pompeji im Nationalmuseum in Neapel, in: T. Ganschow, M. Steinhart et al. (Hg.) Otium. Festschrift für Volker Michael Strocka, Remshalden, B. A. Greiner, 2005, 9-14.

Binsfeld W. Zum Trierer Anaximander-Mosaik. Funde und Ausgrabungen im Bezirk Trier. Aus der Arbeit des Rheinischen Landesmuseums Trier. Kurtrierisches Jahrbuch 1977, 17, 130-131.

Blanck H. Anaximander in Taormina. Mitteilungen des Deutschen Archäologischen Instituts, Römische Abteilung 1997, 104, 507-511.

Blanck H. Zum Anaximander-Relief im Museo Nazionale Romano, in: H.von Steuben (Hg.) Antike Porträts: Zum Gedächtnis von Helga von Heintze. Möhnesee, Bibliopolis, 1999, 47-51.

Brodersen K. Sonnenuhren bei Cetius Faventinus. Gymnasium 2016, 123, 73-84.

Brodersen K. und Chr. (Hgg.) Marcus Cetius Faventinus. Das Römische Eigenheim. De Architectura privata. Lateinisch und Deutsch. Wiesbaden, Marix Verlag, 2015.

Couprie D. L. Heaven and Earth in Ancient Greek Cosmology. From Thales to Heraclides Ponticus. New York u.a., Springer, 2011.

Darsow W. Die Kore des Anaximandros. Jahrbuch des Deutschen Archäologischen Instituts 1954, 69, 101117.

Dorandi T. (ed.) Diogenes Laertios. Lives of Eminent Philosophers. With intr. Cambridge, CUP, 2013.

Ewald B. Chr. Der Philosoph als Leitbild. Ikonographische Untersuchungen an Römischen Sarkophagreliefs. Mainz, Zabern, 1999.

Gaiser K. Das Philosophenmosaik in Neapel. Eine Darstellung der platonischen Akademie. Heidelberg, Carl Winter, 1980.

Hannah R. Time in Antiquity. London - New York, Routledge, 2009.

von den Hoff R. Philosophenporträts des Früh- und Hochhellenismus. München, Biering \& Brinkmann, 1994.

Hoffmann P., Hupe J., Goethert K. Katalog der römischen Mosaike aus Trier und dem Umland. Mainz, Zabern, 1999.

Krüger E. (a) Berichte über die Tätigkeit der Provinzialmuseen in der Zeit vom 1. April 1907 bis 31. März 1908, II. Trier. Bonner Jahrbücher. Jahrbücher des Vereins von Altertumsfreunden im Rheinlande 1909, $118,135-144$.

die einstige Nutzung der zugehörigen Baulichkeiten“ (Dr. Hupe, Rheinisches Landesmuseum Trier, nachrichtlich).

${ }^{46}$ Mikhail Pozdnev regt an, daran zu denken, „dass der Schöpfer des Mosaiks wahrscheinlich die protoi heuretai darzustellen beabsichtigte (der Gnomon ist im Bild zentral; auf den Gegenstand ist die Aufmerksamkeit des Zuschauers gelenkt; m. E. wird hier gerade das Erfindungsmoment, etwa das heureka repräsentiert), und Anaximander ihm eben als solcher galt“.

47 Schefold 1997, 382.

48 Anaximanders Gnomon erweckt jedenfalls immer wieder von Neuem das Interesse der Forschung. So hat jüngst Philip Thibodeau (2017) in einem Beitrag einen Rekonstruktionsversuch unternommen und dabei auf Parallelen zur Augustussonnenuhr in Rom hingewiesen.

49 Wertvolle Hinweise bei der Abfassung des vorliegenden Beitrages erhielt ich von Dirk L. Couprie. Ihm sei herzlich dafür gedankt! 
Krüger E. (b) [Beschreibung des Fundes]. Jahresbericht der Gesellschaft für nützliche Forschungen zu Trier für die Jahre 1906-1908. 1909, NF 1, 16.

Lang J. Mit Wissen geschmückt? Zur bildlichen Rezeption griechischer Dichter und Denker in der römischen Lebenswelt. Wiesbaden, Reichert, 2012.

Mansfeld J., Primavesi O. (Hg.) Die Vorsokratiker, Griechisch / Deutsch. Ausgewählt, übersetzt und erläutert. Stuttgart, Reclam, 2011.

Marcovich M. (ed.) Diogenis Laertii Vitae philosophorum I. Libri I - X. Berlin - New York, De Gruyter, 2008.

Morgan Th. Romano-British Mosaic Pavements: A History of their Discovery and a Record and Interpretation of their Designs. With Plates, Plain and Coloured, of the Most Important Mosaics. London, Whiting \& co., 1886.

Parlasca K. Die Römischen Mosaiken in Deutschland. Berlin, De Gruyter, 1959.

Pattenden P. Sundials in Cetius Faventinus. CQ 1979, 29, 203-212.

Schaldach K. Römische Sonnenuhren. Eine Einführung in die antike Gnomonik. Frankfurt am Main, Verlag Harri Deutsch, ${ }^{3} 2001$.

Schefold K. Die Bildnisse der antiken Dichter, Redner und Denker. Basel, Schwabe, 1997.

Severino N. Pelecinum o Pelignum? Note sull' identificazione di due antichi orologi solari. http://www.gnomonica.it/pelignum.html (16.10.2018)

Thibodeau P. Anaximander's Spartan Sundial. CQ 2017, 67, 374-379.

Traversari G. Il „Pelecinum“ - Un particolare tipo di orologio solare raffigurato su alcuni rilievi di sarcofagi di età romana, in: G. Romano, G. Traversari (eds). Colloquio Internazionale Archeologia e Astronomia, Venedig 3. - 6. Mai 1989. Roma, Giorgio Bretschneider, 1991, 66-73.

Winter E. Zeitzeichen: Zur Entwicklung und Verwendung antiker Zeitmesser. Berlin - Boston, De Gruyter, 2013.

Wöhrle G. (Hg.) Die Milesier: Anaximander und Anaximenes (mit Beiträgen von Oliver Overwien). Traditio Praesocratica 2. Berlin, De Gruyter, 2012.

\section{Anhang: Abbildungen}

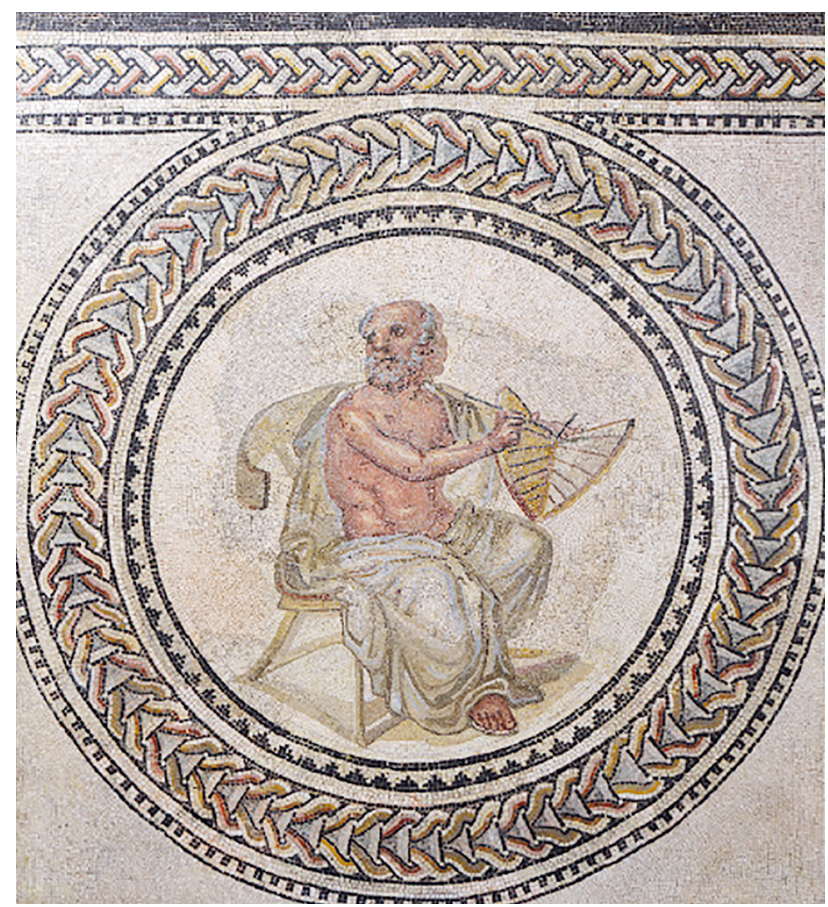

Abb. 1. Anaximander - Mosaik Trier, Rheinisches Landesmuseum Trier 


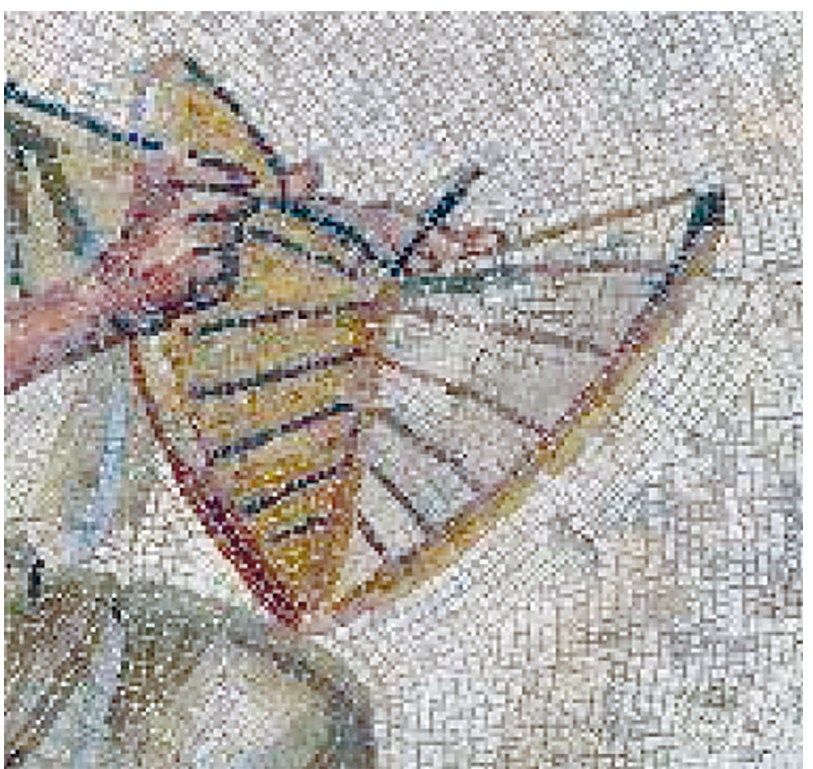

Abb. 2. Anaximander - Mosaik Trier Detail

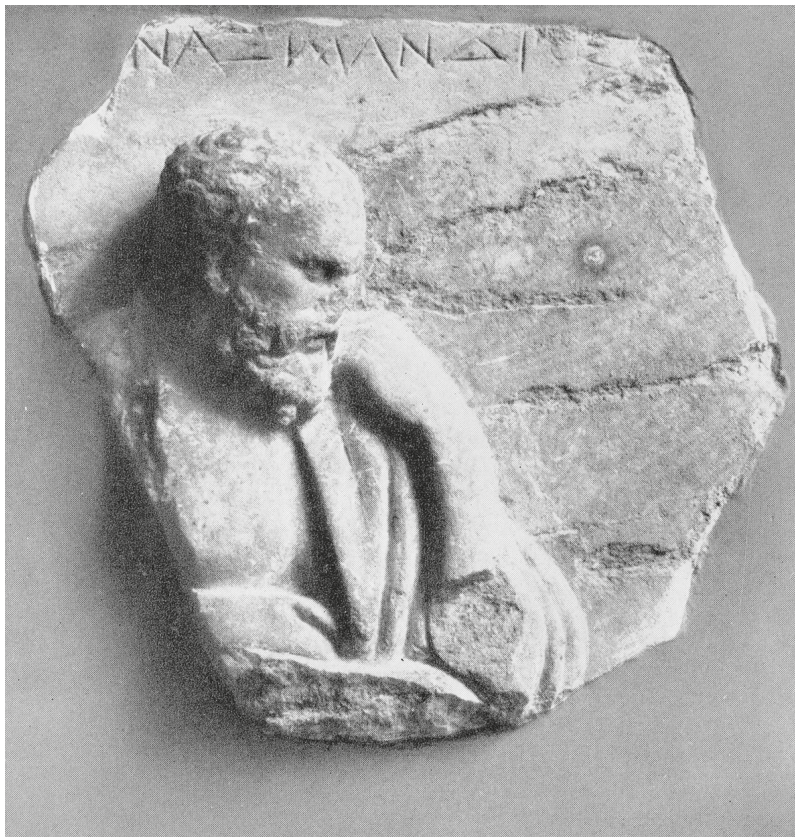

Abb. 3. Anaximanderrelief, Rom, Thermenmuseum, Inv. 506, aus Schefold 1997, Abb. 310 


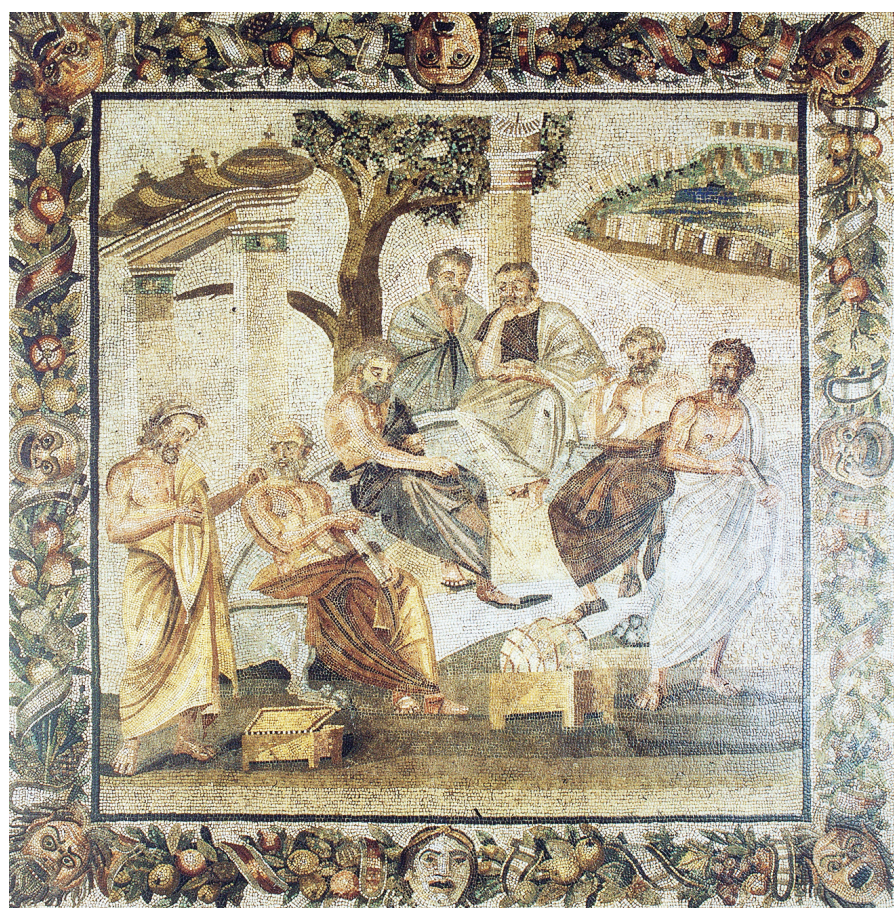

Abb. 4. Philosophenmosaik aus Pompeji, Neapel, Nationalmuseum, aus Andreae 2005, 13, Abb. 2

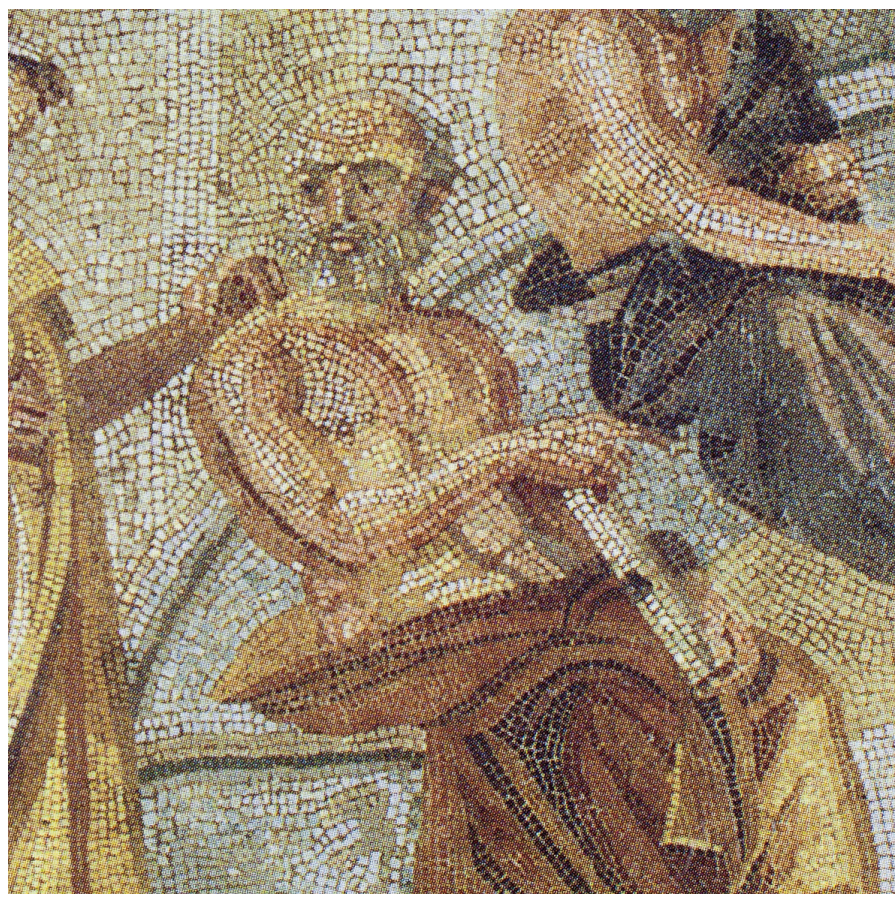

Abb. 5 Philosophenmosaik aus Pompeji, Ausschnitt 


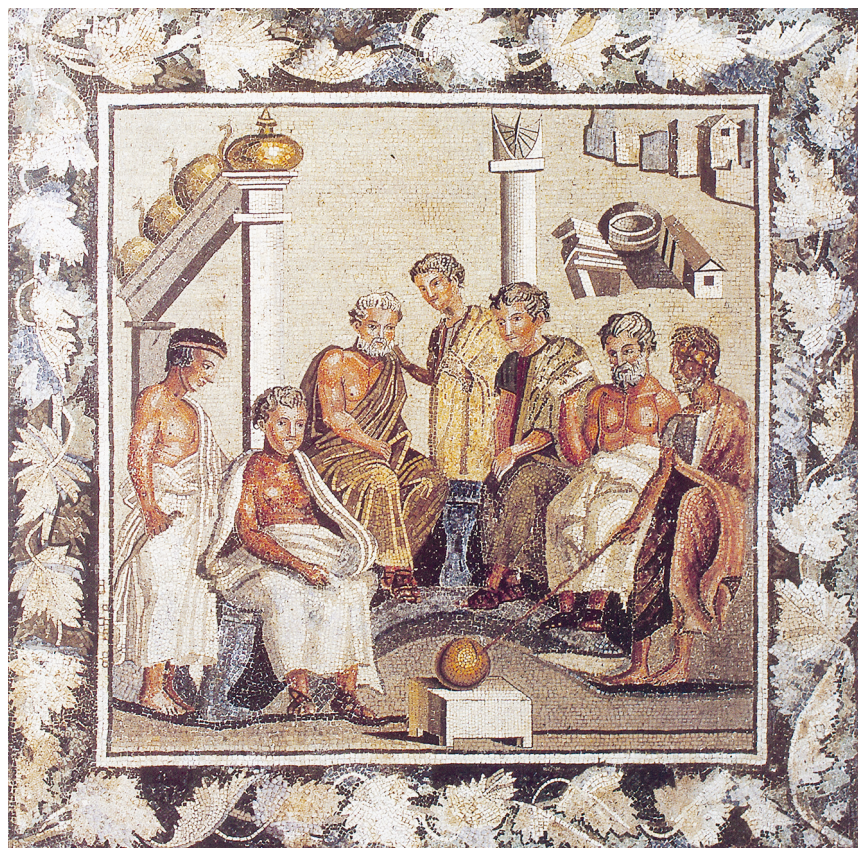

Abb. 6. Mosaik der Sieben Weisen aus Sarsina, Rom, Villa Albani, aus Andreae 2005, 12, Abb. 1

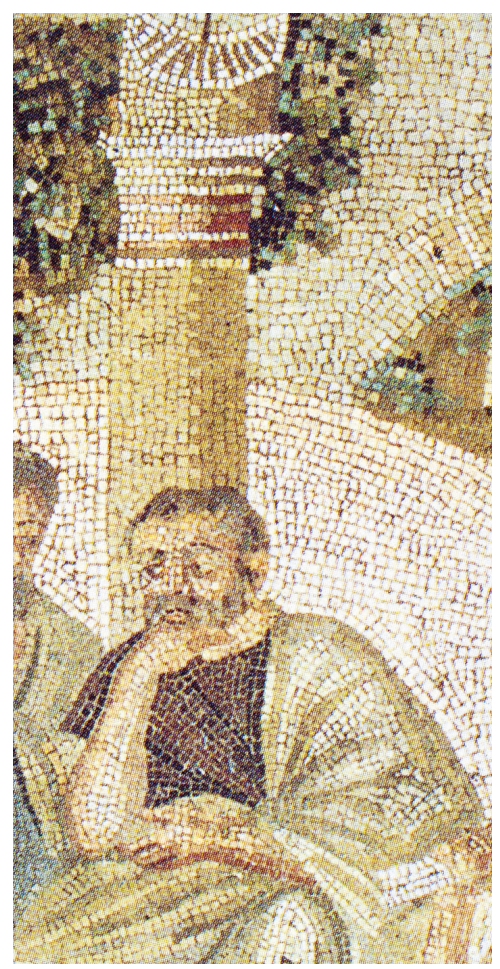

Abb. 7. Philosophenmosaik aus Pompeji, Ausschnitt 
Анаксимандр, Патрокл или, быть может, Эвдокс?

Краткая история интерпретации т. н. «Анаксимандровой мозаики» на фоне биографических, доксографических и археологических свидетельств

\section{Георг Вёрле}

Трирский университет,

Университетсринг 156 54286, Трир, Германия; woehrle@uni-trier.de

Датируемый II в. н.э. мозаичный портрет, осколок многофигурной композиции, обнаруженный в 1898 г. при раскопках римского дома в Трире и хранящийся в Rheinisches Landesmuseum, выделяется превосходной сохранностью, не ремесленным качеством исполнения и нетривиальностью образа: лысый, седобородый философ развернут вполоборота, смотрит вправо вверх, а в руках держит предмет, ближе всего напоминающий солнечные часы. Догадаться нетрудно, и уже в первой публикации (1909 г.) тогдашний директор Landesmuseum Эмиль Крюгер предположил, что изображен Анаксимандр. Таким было и остается общее мнение. Впрочем, голоса скептиков также слышны: скудость сведений об Анаксимандре позволяла соотнести трирского философа со скульптором Патроклом, создателем pelecinum (так у Витрувия) - особого вида солнечных часов, сходного с тем, что на мозаике. Однако малоизвестная фигура едва ли вдохновила бы художника. Более уместной кандидатурой представляется Евдокс, ученый известнее даже Анаксимандра и также сконструировавший гномон (arachne, согласно Витрувию: пусть эти часы и другой формы, едва ли автор мозаики стремился к историко-научной достоверности). Можно долго и пристально рассматривать фигуру, отождествляемую с Евдоксом на помпейской «мозаике философов», предполагаемое изображение Анаксимандра на ней же, известный римский «рельеф Анаксимандра» (с надписью!), а равно и другие близкие по типу изображения - надежной атрибуции эти сопоставления не дают. История рецепции Анаксимандра античной культурой не противоречит распространенному взгляду: он и никто другой должен был восприниматься позднеримским искусством как изобретатель гномона. Будь у нас другие фрагменты мозаики, возможно, мы увидели бы на них других изобретателей. В любом случае сомнительным кажется мнение, будто бы философ, обращая взгляд к небу, мыслит «о возникновении и уничтожении небесных тел» (К.Шефолд). Проще думать, что изобретатель солнечных часов следит за солнцем.

Ключевые слова: Анаксимандр, трирская мозаика, Евдокс, скульптор Патрокл, изображения античных философов. 